PROCEEDINGS OF THE

AMERICAN MATHEMATICAL SOCIETY

Volume 134, Number 3, Pages 917-920

S 0002-9939(05)08012-3

Article electronically published on July 19, 2005

\title{
AN OBSERVATION ON REALCOMPACT SPACES
}

\author{
Z. ERCAN
}

(Communicated by Joseph A. Ball)

\begin{abstract}
We give a characterization of realcompact spaces in terms of nets. By using the technique of this characterization we give easy proofs of the Tychonoff Theorem and the Alaoglu Theorem.
\end{abstract}

Let $X$ be Tychonoff space and let $\left(x_{i}\right)$ be a net in $X$. It is clear that $\left(x_{i}\right)$ converges if $\lim f\left(x_{i}\right)=f(x)$ for each real-valued continuous function $f$ on $X$ for some $x \in X$. This motivates the following question: Does $\left(x_{i}\right)$ converge if $\lim f\left(x_{i}\right)$ exists for each continuous real-valued function $f$ on $X$ ? We will show that this characterizes realcompact spaces. We believe that this characterization may be of interest because of its simplicity and accessibility.

Throughout this paper we suppose that the topological spaces are Hausdorff. As usual $C(X)$ stands for the algebra of real-valued continuous functions on a topological space $X$ under the pointwise operations; that is, for each $f, g, h \in C(X)$ and $\alpha \in \mathbb{R}$,

$$
(f+g)(x)=f(x)+g(x), \quad(f g)(x)=f(x) g(x) \quad \text { and } \quad(\alpha f)(x)=\alpha f(x) .
$$

A map $\varphi: C(X) \longrightarrow \mathbb{R}$ is called an (algebra) homomorphism if

$$
\varphi(f g+\alpha h)=\varphi(f) \varphi(g)+\alpha \varphi(h)
$$

for each $f, g, h \in C(X)$ and $\alpha \in \mathbb{R}$. In [8] it is shown that a map $\varphi: C(X) \longrightarrow \mathbb{R}$ is a homomorphism if and only if it is a ring homomorphism; that is,

$$
\varphi(f g+h)=\varphi(f) \varphi(g)+\varphi(h)
$$

for each $f, g, h \in C(X)$. The subalgebra of bounded functions in $C(X)$ is denoted by $C_{b}(X)$. We refer to [7] for more details on $C(X)$ and $C_{b}(X)$ algebras.

Recall that a topological space $X$ is called Tychonoff if for every $x \in X$ and every closed set $F \subset X$ such that $x \notin F$ there exists a bounded function $f$ in $C(X)$ such that $f(x)=0$ and $f(y)=1$ for $y \in F$. A topological space $X$ is called a realcompact space if it is homeomorphic to a closed subspace of the product space $\prod_{i \in I} \mathbb{R}$ for some index set $I$. It is well known that a Tychonoff space $X$ is a realcompact space if and only if for each non-zero homomorphism $\pi: C(X) \longrightarrow \mathbb{R}$ there exists $x_{\pi} \in X$ such that $\pi(f)=f\left(x_{\pi}\right)$ for each $f \in C(X)$ (an elementary proof of this without using of Axiom of Choice can be found in a recent paper [6]). Each Tychonoff space $X$ can be embedded into a unique realcompact space $v X$ as a dense subspace such that $C(X)$ and $C(v X)$ are algebraic isomorphic (see [5, p. $218])$. For each $f \in C(X), f^{r} \in C(v X)$ denotes the image of $f$. If $\pi: C(X) \longrightarrow \mathbb{R}$,

Received by the editors September 17, 2004 and, in revised form, October 13, 2004.

2000 Mathematics Subject Classification. Primary 54D60.

(C)2005 American Mathematical Society 
then $\pi^{r}: C(v X) \longrightarrow \mathbb{R}$ is defined by $\pi^{r}(g)=\pi(h)$, where $h$ is the restriction of $g$ to $X$. This observation leads to the following lemma.

Lemma 1. Let $X$ be a Tychonoff space and let $\pi: C(X) \longrightarrow \mathbb{R}$ be a nonzero homomorphism. Then there exists a net $\left(x_{i}\right)$ in $X$ such that $\pi(f)=\lim f\left(x_{i}\right)$ for each $f \in C(X)$.

Proof. Let $\pi: C(X) \longrightarrow \mathbb{R}$ be a nonzero homomorphism. As the algebra $C(X)$ can be identified with $C(v X)$, where $v X$ is defined above, there exists $a \in v X$ and a net $\left(x_{i}\right) \in X$ with $x_{i} \longrightarrow a$ such that

$$
\pi(f)=\pi^{r}\left(f^{r}\right)=f^{r}(a)=\lim f^{r}\left(x_{i}\right)=\lim f\left(x_{i}\right) .
$$

This completes the proof.

Now we are ready to present the main result as follows.

Theorem 2. Let $X$ be a Tychonoff space. Then the following are equivalent.

i. $X$ is a realcompact space.

ii. A net $\left(x_{i}\right)$ in $X$ converges if and only if limf $\left(x_{i}\right)$ exists for each $f \in C(X)$.

Proof. Suppose that $\mathbf{i}$ holds and $\left(x_{i}\right)$ is a net in $X$ such that $\lim f\left(x_{i}\right)$ exists for each $f \in C(X)$. Let

$$
\pi: C(X) \longrightarrow \mathbb{R}, \quad \pi(f)=\lim f\left(x_{i}\right) .
$$

Then $\pi$ is a nonzero homomorphism. As $X$ is a realcompact space there exists an $x \in X$ such that $\pi(f)=f(x)$. Since $X$ is a Tychonoff space and $\lim f\left(x_{i}\right)=f(x)$ for each $f \in C(X),\left(x_{i}\right)$ converges to $x$. Now suppose that ii holds and $\pi: C(X) \longrightarrow \mathbb{R}$ is a nonzero homomorphism. From the above lemma there exists a net $\left(x_{i}\right)$ in $X$ such that $\pi(f)=\lim f\left(x_{i}\right)$ for each $f \in C(X)$. From the hypothesis, $x_{i} \rightarrow x$, so $\pi(f)=f(x)$ for each $f \in C(X)$. Hence $X$ is a realcompact space.

In [10], a net $\left(x_{i}\right)$ of points of a topological space $X$ is called a $C(X)$-net in $X$ if for every real-valued continuous function $f$ on $X$ the net $\left(f\left(x_{i}\right)\right)$ is convergent. A topological space $X$ is $C$-complete whenever every $C(X)$-net in $X$ has a cluster point in $X$. In [10] it is observed that every realcompact space is $C$-complete. For a Tychonoff space $X$, from the above theorem, the proof of the corollary below is easy.

Corollary 3. Let $X$ be a Tychonoff space. Then the following are equivalent.

i. $X$ is a realcompact space.

ii. $X$ is $C$-complete.

iii. Each $C(X)$-net is convergent.

In general the construction of the Stone-Čech compactification of a Tychonoff space is given by using the Tychonoff Theorem. There is another way of doing this construction, that is as follows: Let $X$ be a Tychonoff space and $\beta X$ be the set of homomorphisms $\pi: C_{b}(X) \longrightarrow \mathbb{R}$ with $\pi \mathbf{1}=1$. Then by using elementary arguments it is not difficult to show that (without using the Tychonoff Theorem) $\beta X$ is a compact Hausdorff space such that $\pi_{\alpha} \longrightarrow \pi$ in $\beta X$ if and only if $\pi_{\alpha}(f) \longrightarrow$ $\pi(f)$ for each $f \in C_{b}(X)$ (see [4]). $\left\{\{\pi: \pi(f) \neq 0\}: f \in C_{b}(X)\right\}$ is a base for the topological space $\beta X$. Under the map $x \longrightarrow \pi_{x}, \pi_{x}(f)=f(x)$, $X$ is dense in $\beta X$, and $C_{b}(X)$ and $C(\beta X)$ are algebraic isomorphic under the operator $T: C_{b}(X) \longrightarrow C(\beta X), T(f)(\pi)=\pi(f)$. By using an argument similar to the above 
theorem and the construction of $\beta X$ as in the above, for a Tychonoff space $X$ the proof of the next theorem is clear.

Theorem 4. Let $X$ be a Tychonoff space. Then the following are equivalent.

i. $X$ is compact.

ii. A net $\left(x_{i}\right)$ in $X$ converges if and only if $\lim f\left(x_{i}\right)$ exists for each $f \in C_{b}(X)$.

One of the important theorems of functional analysis is the Alaoglu Theorem (see [1] for a proof). The known proof of this theorem depends on the Tychonoff Theorem. By using the above theorem we can give an alternative and easy proof (depending on the Stone-Čech compactification and without using the Tychonoff Theorem) of this theorem as follows.

Theorem 5 (Alaoglu, [1). Let $X$ be a locally convex space and let $V$ be a neighborhood of zero. Then the polar $V^{\circ}$ is $\sigma\left(X^{\prime}, X\right)$-compact.

Proof. It is clear that $V^{\circ}$ is a $\sigma\left(X^{\prime}, X\right)$-Tychonoff space. Let $\left(f_{\alpha}\right)$ be a net in $V^{\circ}$ such that $\lim \pi\left(f_{\alpha}\right)$ exists for each real-valued bounded continuous function on $V^{\circ}$. Then in particular $\lim \pi_{x}\left(f_{\alpha}\right)$ exists for each $x \in X$, where $\pi_{x}: V^{\circ} \longrightarrow \mathbb{R}$, $\pi_{x}(f)=f(x)$. Let $f: X \longrightarrow \mathbb{R}$ be defined by $f(x)=\lim \pi_{x}\left(f_{\alpha}\right)$. Now it is clear that $f \in V^{\circ}$ and $f_{\alpha} \longrightarrow f$ in $\sigma\left(X^{\prime}, X\right)$. Now from Theorem $4, V^{\circ}$ is $\sigma\left(X^{\prime}, X\right)$ compact.

The classical Tychonoff Theorem states that the product space $X$ of compact Hausdorff spaces $\left(X_{\alpha}\right)$ is also compact. In the literature there are many different proofs of this theorem (see [3], 9]). By using Theorem 3 we can give an alternative and easy proof of this as follows:

Theorem 6 (Tychonoff Theorem). Let $\left\{X_{\alpha}: \alpha \in \Gamma\right\}$ be a set of compact Hausdorff spaces. Then the product space $X=\prod_{\alpha} X_{\alpha}$ is compact.

Proof. Clearly $X$ is a Tychonoff space. For each $\alpha_{0} \in \Gamma$, let $P_{\alpha_{0}}: X \longrightarrow X_{\alpha_{0}}$ be defined by $P_{\alpha_{0}}\left(\left(x_{\alpha}\right)\right)=x_{\alpha_{0}}$. Let $x=\left(\left(x_{i}^{\alpha}\right)_{\alpha}\right)_{i}$ be a net in the product space $X$ such that $\left(F\left(\left(x_{i}^{\alpha}\right)_{\alpha}\right)\right)_{i}$ is convergent for each real-valued bounded continuous function $F$ on $X$. Then for each $\alpha_{0}$ and each bounded function $f \in C\left(X_{\alpha_{0}}\right),\left(f\left(x_{i}^{\alpha_{0}}\right)\right)=$ $\left(f \circ P_{\alpha_{0}}(x)\right)$ is convergent. As $X_{\alpha_{0}}$ is compact, from Theorem $4, x_{i}^{\alpha_{0}} \longrightarrow x^{\alpha_{0}}$ for some $x^{\alpha_{0}} \in X_{\alpha_{0}}$. Let $x_{\infty}=\left(x^{\alpha}\right)$. Then $x$ is convergent to $x_{\infty}$ in $X$. Again from Theorem $4, X$ is compact.

\section{REFERENCES}

[1] L. Alaoglu, Weak topologies of normed linear spaces, Ann. of Math. (2) 41,(1940), 252-267. MR0001455 (1:241e)

[2] C. D. Aliprantis and O. Burkinshaw, Positive operators, Pure and Applied Mathematics, 119. Academic Press, Inc., Orlando, FL, 1985. MR0809372 (87h:47086)

[3] P. R. Chernoff, A simple proof of Tychonoff's theorem via nets, Amer. Math. Monthly 99 (1992), no. 10, 932-934. MR.1190558 (93m:54042)

[4] E. de Jonge and A. C. M. van Rooij, Introduction to Riesz spaces, Mathematical Centre Tracts, No. 78. Mathematisch Centrum, Amsterdam, 1977. MR0473777 (57:13439)

[5] R. Engelking, General Topology, Heldermann Verlag Berlin, 1988. MR.1039321 (91c:54001)

[6] Z. Ercan and S. Onal, A remark on the homomorphism on $C(X)$, Proc. Amer. Math. Soc. (to appear).

[7] L. Gillman and M. Jerison, Rings of continuous functions, New York, 1960. MR0116199 $(22: 6994)$ 
[8] L. E. Pursell, comment: "Homomorphisms on $C(\mathbb{R})$ ", Amer. Math. Monthly 94 (1987), no.7, 646. MR0935848 (89b:46065)

[9] D. G. Wright, Tychonoff's theorem, Proc. Amer. Math. Soc. 120 (1994), no. 3, 985-987. MR.1170549 (94e:54032)

[10] H. J. Wu, A generalized process of Stone-Cech compactification and realcompactification of an arbitrary topological space, General topology and applications (Middletown, CT, 1988), Lecture Notes in Pure and Appl. Math., 1990(123) 269-283. MR.1057644 (91h:54039)

Department of Mathematics, Middle East Technical University, 06531 Ankara, TURKEY

E-mail address: zercan@metu.edu.tr 